\title{
Método de elementos finitos para el cálculo del campo electromagnético alrededor de una línea de transmisión eléctrica
}

\author{
Finite element method for calculating the electromagnetic \\ field around an electrical transmission line
}

\author{
Raúl Sanhueza H. ${ }^{1 *} \quad$ Ildefonso Harnisch V. ${ }^{1} \quad$ Sebastián Rojo A. ${ }^{1}$ \\ Recibido 4 de Diciembre de 2020, aceptado 28 de Diciembre de 2020 \\ Received: December 4, 2020 Accepted: December 28, 2020
}

\begin{abstract}
RESUMEN
En este trabajo se presenta la aplicación del método de elementos finitos (MEF) para cuantificar el campo electromagnético que se irradia alrededor de una línea de transmisión eléctrica. Para este efecto, el desarrollo de las ecuaciones diferenciales de segundo grado que describen el comportamiento físico de los campos electromagnéticos se realizó en dos dimensiones, considerando que los conductores de la línea de transmisión son infinitamente largos y rectos. Como aplicación se presentan resultados de simulación a distintas configuraciones de líneas, en condiciones de operación normal. De los resultados se puede concluir que, para configuraciones estándar de líneas, el efecto electromagnético asociado no parece relevante, sin embargo, el uso de las líneas de energía dentro de zonas urbanas motiva la necesidad de cuantificar estos efectos, en virtud del cumplimiento de normas de emisión de campos electromagnéticos de baja frecuencia.
\end{abstract}

Palabras clave: Campo electromagnético, método de elementos finitos (MEF), líneas de transmisión.

\begin{abstract}
This article presents the finite element method (FEM) application to quantify the electromagnetic field radiating around an electrical transmission line. For this purpose, the development of second-degree differential equations describing the physical behavior of electromagnetic fields was performed in two dimensions, considering that the conductors of the transmission line are infinitely long and straight. As an application simulation, results are presented to different line configurations under normal operating conditions. From the results, it can be concluded that, for standard line configurations, the associated electromagnetic effect does not seem relevant; however, the use of power lines within urban areas motivates the need to quantify these effects under compliance low-frequency electromagnetic field emission standards.
\end{abstract}

Keywords: Electromagnetic field, finite element method (FEM), transmission lines.

\footnotetext{
1 Universidad de Tarapacá. Departamento de Ingeniería Eléctrica y Electrónica. Arica, Chile.

E-mail: sanhueza@uta.cl; harnisch@uta.cl

* Autor de correspondencia: sanhueza@uta.cl
} 


\section{INTRODUCCIÓN}

La comunidad en general se encuentra cada vez más expuesta a campos electromagnéticos, debido al uso generalizado y extendido de la electricidad, donde sus efectos pueden traer graves problemas al medio ambiente y para la salud y seguridad de las personas, o de la población, debido a exposiciones frecuentes o permanentes. Por este motivo, desde hace algunas décadas, existe una gran preocupación por parte de la comunidad científica y medio ambiental, acerca del posible efecto perjudicial de los campos electromagnéticos provenientes de las líneas de transmisión, y en particular de las líneas de extra alta tensión que más merecen atención en este sentido $[1,2,3]$.

En el proyecto de una línea de transmisión, los campos electromagnéticos son una importante limitación y resulta necesario justificar sus diferentes valores. Entre los campos que necesitan más atención está el campo eléctrico en la superficie del conductor, el cual una vez pasado de su límite, puede producir el denominado efecto corona y traer grandes pérdidas de transmisión. Otro problema que se puede encontrar es el efecto del campo electromagnético a ras de suelo, lo que provoca graves problemas en la banda de paso de la línea de transmisión y sus alrededores. Así, en definitiva, en el estudio de una línea de transmisión el campo electromagnético termina por interferir en la elección de las estructuras, cables, conductores, en la banda de paso y en el mismo trazado. Por lo tanto, los estudios relacionados con los campos electromagnéticos generados por líneas de transmisión son de una gran importancia $[4,10]$.

El empleo de líneas de alta tensión dentro de zonas urbanas es un problema presente en todas las grandes ciudades. La compactación de líneas de transmisión de $115 \mathrm{kV}$ y $66 \mathrm{kV}$ se justifica tanto por una considerable economía en la inversión como por el uso cada vez mayor de estos niveles de tensión como voltaje secundario en las zonas urbanas y suburbanas. Es por esto que el derecho de paso, los factores ambientales y los límites de los campos se convierten en factores muy importantes, [4].

El Método de los elementos finitos (MEF) es uno de los métodos numéricos más populares para la simulación por computador $[5,6]$. La ventaja principal del MEF, sobre otros métodos numéricos en aplicaciones técnicas, es la capacidad de manejar problemas de geometría circular y no lineal dependiente del tiempo [6]. Por lo tanto, este método, es conveniente para resolver el problema de los efectos del campo magnético alrededor de una línea de transmisión, causada por una sección circular de conductores de alta tensión. Especialmente, cuando la magnitud de los campos debe ser cuidadosamente determinada en un delimitado dominio o región [6, 7].

Aunque los métodos convencionales sean más simples que el uso del MEF, se ven limitados a un sistema de geometría simple [8]. En la práctica, varias estructuras metálicas pueden ser encontradas en la línea debajo de la torre de acero, como ser, líneas de comunicación cercanas, u otras líneas de paso con voltaje inferior. La utilización del MEF incluye estos efectos seleccionando el material para cada sistema de dominios adicionales. Con este hecho, el MEF es una de las potenciales herramientas de simulación numérica para analizar problemas de campo magnético de regiones de materiales combinados [9].

Este trabajo utiliza el método de elementos finitos, para desarrollar un modelo que describe el comportamiento físico del campo electromagnético en los alrededores de una línea eléctrica. Así, de la resolución de las ecuaciones diferenciales de segundo grado resultantes del modelo, es posible cuantificar el campo electromagnético en los alrededores de la línea y, en particular, en cualquier punto en la cercanía de la línea que pueda resultar de interés.

\section{MODELO MATEMÁTICO DEL CAMPO ELECTROMAGNÉTICO DE UNA LÍNEA DE TRANSMISIÓN}

En el estudio de la propagación de las ondas electromagnéticas, la forma diferencial de las ecuaciones de Maxwell es la más utilizada [6].

$$
\begin{gathered}
\nabla \times E=-\frac{\partial B}{\partial t} \\
\nabla \times H=J+\frac{\partial D}{\partial t} \\
\nabla \cdot D=\rho \\
\nabla \cdot B=0
\end{gathered}
$$


Donde:

E: Intensidad del campo eléctrico en $(\mathrm{V} / \mathrm{m})$.

D: Densidad de flujo eléctrico $\left(\mathrm{A} / \mathrm{m}^{2} \mathrm{~s}\right)$.

$\mathrm{H}$ : Intensidad del campo magnético en $(\mathrm{A} / \mathrm{m})$.

B: Densidad de flujo magnético (T).

J: Densidad de corriente $\left(\mathrm{A} / \mathrm{m}^{2}\right)$.

$\rho:$ Densidad de carga eléctrica $\left(\mathrm{C} / \mathrm{m}^{2}\right)$.

También, para el planteamiento de la ecuación de onda se puede introducir las ecuaciones para un medio lineal isotrópico y homogéneo.

$$
\begin{aligned}
& D=\varepsilon E \\
& B=\mu H \\
& J=\sigma E
\end{aligned}
$$

Con esto la ecuación de onda o de Helmholtz para el campo electromagnético alrededor de una línea se presenta en dos formas:

$$
\begin{gathered}
\nabla^{2} \times E=\mu \sigma \frac{\partial E}{\partial t}+\mu \varepsilon \frac{\partial^{2} E}{\partial t^{2}} \\
\nabla^{2} \times H=\mu \sigma \frac{\partial H}{\partial t}+\mu \varepsilon \frac{\partial^{2} H}{\partial t^{2}}
\end{gathered}
$$

Donde:

$\mu$ : Permeabilidad magnética del medio $(\mathrm{H} / \mathrm{m})$

$\varepsilon: \quad$ Permitividad eléctrica del medio $(\mathrm{F} / \mathrm{m})$

Estas ecuaciones al ser analizadas como campos harmónicos oscilando a una frecuencia constante, permiten aproximar las derivadas con una notación compleja, $\frac{\partial^{2}}{\partial t^{2}}=\omega^{2}, \frac{\partial}{\partial t}=j \omega$

De esta manera, las ecuaciones (8) y (9) quedan expresadas en términos de los vectores de campo de forma fasorial como sigue:

$$
\begin{aligned}
& \nabla^{2} \times E=j \omega \mu(\sigma+j \omega \varepsilon) E \\
& \nabla^{2} \times H=j \omega \mu(\sigma+j \omega \varepsilon) H
\end{aligned}
$$

Finalmente, al considerar el problema en dos dimensiones, para el plano cartesiano $(\mathrm{x}, \mathrm{y})$, se tiene que:

$$
\frac{\partial}{\partial x}\left(\frac{1}{\mu} \frac{\partial E}{\partial x}\right)+\frac{\partial}{\partial y}\left(\frac{1}{\mu} \frac{\partial E}{\partial y}\right)-\left(j \omega \sigma-\omega^{2} \varepsilon\right) E=0
$$

$$
\frac{\partial}{\partial x}\left(\frac{1}{\mu} \frac{\partial H}{\partial x}\right)+\frac{\partial}{\partial y}\left(\frac{1}{\mu} \frac{\partial H}{\partial y}\right)-\left(j \omega \sigma-\omega^{2} \varepsilon\right) H=0
$$

En consecuencia, la ecuación (12) y (13) representa el modelo matemático del campo electromagnético en los alrededores de una línea de transmisión.

\section{MEF APLICADO A UNA LÍNEA DE TRANSMISIÓN}

\section{A. Discretización}

En el empleo de MEF, el área transversal a una línea es discretizada mediante elementos finitos triangulares de primer grado [11]. Como ejemplo, se presenta el área de trabajo (40 m x $47 \mathrm{~m}$ ) para una línea de $500 \mathrm{kV}$ en circuito simple. Esta área está discretizada con 3943 elementos y 2040 nodos.

\section{B. Formulación del modelo mediante elementos finitos}

La magnitud del campo eléctrico y del campo magnético en cualquier punto de coordenadas ( $\mathrm{x}$, y) en el área de trabajo se expresa como:

$E(x, y)=E_{1} N_{1}(x, y)+E_{2} N_{2}(x, y)+E_{3} N_{3}(x, y)(14)$

$$
H(x, y)=H_{1} N_{1}(x, y)+H_{2} N_{2}(x, y)+H_{3} N_{3}(x, y)
$$

Donde, $\mathrm{E}_{1}, \mathrm{E}_{2}, \mathrm{E}_{3}, \mathrm{H}_{1}, \mathrm{H}_{2}, \mathrm{yH}$. de las magnitudes de campo eléctrico y magnético en cada uno de los vértices del elemento triangular en el cual está contenido el punto (x,y). $\mathrm{N}_{1}, \mathrm{~N}_{2} \mathrm{y}$ $\mathrm{N}_{3}$, son funciones de forma para para este elemento, que toma valores de acuerdo a:

$$
N_{1}(x, y)=\frac{a_{1}+b_{1} x+c_{1} y}{2 \Delta}
$$
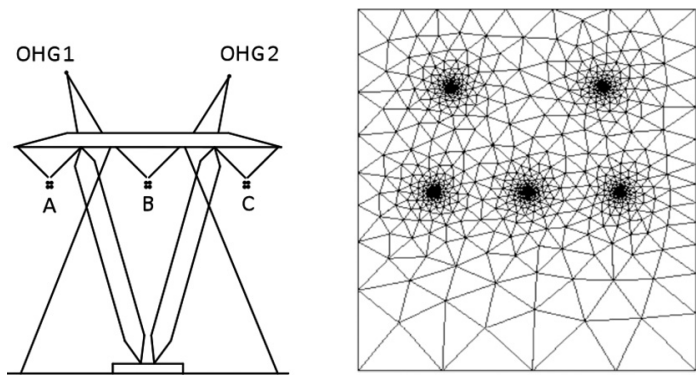

Figura 1. Sistema de transmisión de $500 \mathrm{kV}$ en circuito simple y su discretización. 


$$
\begin{aligned}
& N_{2}(x, y)=\frac{a_{2}+b_{2} x+c_{2} y}{2 \Delta} \\
& N_{3}(x, y)=\frac{a_{3}+b_{3} x+c_{3} y}{2 \Delta}
\end{aligned}
$$

Donde, $\Delta$ es el área de cada elemento triangular y el valor de las constantes $\mathrm{a}_{\mathrm{i}}, \mathrm{b}_{\mathrm{i}} \mathrm{y} \mathrm{c}_{\mathrm{i}}$ está dado por las coordenadas de cada vértice del elemento triangular, de manera que:

$$
\begin{aligned}
& \Delta=\frac{1}{2} \operatorname{det}\left[\begin{array}{lll}
x_{1} & y_{1} & 1 \\
x_{2} & y_{2} & 1 \\
x_{3} & y_{3} & 1
\end{array}\right] \\
& a_{1}=x_{2} y_{3}-x_{3} y_{2} \quad b_{1}=y_{2}-y_{3} \quad c_{1}=x_{3}-x_{2} \\
& a_{2}=x_{3} y_{1}-x_{1} y_{3} \quad b_{2}=y_{3}-y_{1} \quad c_{2}=x_{1}-x_{3} \\
& a_{3}=x_{1} y_{2}-x_{2} y_{1} \quad b_{3}=y_{1}-y_{2} \quad c_{3}=x_{2}-x_{1}
\end{aligned}
$$

Así entonces, aplicando el método de los residuos ponderados con el enfoque de Galerkin a las ecuaciones diferenciales (12) y (13), donde las integraciones se realizan sobre los elementos de dominio $\Omega$, se obtiene.

$$
\begin{aligned}
& \frac{1}{\mu} \int_{\Omega} w\left(\frac{\partial}{\partial x}\left(\frac{\partial E}{\partial x}\right)+\frac{\partial}{\partial y}\left(\frac{\partial E}{\partial y}\right)\right) d \Omega-\left(j \omega \sigma-\omega^{2} \varepsilon\right) \int_{\Omega} w E d \Omega=0 \\
& \frac{1}{\mu} \int_{\Omega} w\left(\frac{\partial}{\partial x}\left(\frac{\partial H}{\partial x}\right)+\frac{\partial}{\partial y}\left(\frac{\partial H}{\partial y}\right)\right) d \Omega-\left(j \omega \sigma-\omega^{2} \varepsilon\right) \int_{\Omega} w H d \Omega=0
\end{aligned}
$$

De manera compacta en notación matricial:

$$
\begin{aligned}
& {[K+M] E=0} \\
& {[K+M] H=0}
\end{aligned}
$$

Donde:

$$
\begin{gathered}
{[K]=\frac{1}{\mu} \int_{\Omega}\left(\frac{\partial w}{\partial x} \frac{\partial E}{\partial x}+\frac{\partial w}{\partial y} \frac{\partial E}{\partial y}\right) d \Omega=} \\
\frac{1}{4 \mu \Delta}\left[\begin{array}{ccc}
b_{1} b_{1}+c_{1} c_{1} & b_{1} b_{2}+c_{1} c_{2} & b_{1} b_{3}+c_{1} c_{3} \\
b_{1} b_{2}+c_{1} c 2 & b_{2} b_{2}+c_{2} c_{2} & b_{2} b_{3}+c_{2} c_{3} \\
b_{1} b_{3}+c_{1} c_{3} & b_{2} b_{3}+c_{2} c_{3} & b_{3} b_{3}+c_{3} c_{3}
\end{array}\right]
\end{gathered}
$$

$$
\begin{aligned}
& {[M]=-\left(j \omega \sigma-\omega^{2} \varepsilon\right) \int_{\Omega} w E d \Omega} \\
& =\frac{\left(j \omega \sigma-\omega^{2} \varepsilon\right) \Delta}{12}\left[\begin{array}{lll}
2 & 1 & 1 \\
1 & 2 & 1 \\
1 & 1 & 2
\end{array}\right]
\end{aligned}
$$

Finalmente, para la magnitud de campo magnético, se deducen las mismas matrices.

\section{Condiciones de borde y parámetros de simulación} El estudio de la MEF generado por líneas de transmisión es muy genérico, pudiéndose analizar el comportamiento de MEF para diferentes configuraciones y condiciones de operación. En este trabajo, se modelaron configuraciones simples en condiciones de operación normal. Las hipótesis consideradas en cada caso son:

- Conductores perfectamente nivelados uno respecto de otro y tierra.

- El campo se calcula para una sección transversal de la línea considerando una altura mínima de los conductores.

- Las fases se consideran como sección circular, la separación del haz es despreciable en comparación con las distancias en las que se calcularon la MEF (1 m por sobre el suelo).

- En todos los casos estudiados se utilizó un margen de 5\% por sobre el voltaje nominal.

- Las corrientes de línea se consideraron de acuerdo al límite térmico de los conductores de fase.

- Las condiciones de borde aplicadas para el problema son, campo magnético y eléctrico nulos a nivel del suelo y en los cables de guardia.

- Para los conductores de fase las condiciones de borde en la periferia se calcularán según la ley de Ampere y ley de Gauss para el campo magnético y el campo eléctrico, respectivamente.

- Al mismo tiempo, la conductividad, permisividad y permeabilidad en el aíre son $\sigma=5,5 \times 10^{-15}$ $(\mathrm{S} / \mathrm{m}), \mu=4 \pi \times 10^{-7}(\mathrm{H} / \mathrm{m})$ y $\mu=8,85 \times 10^{-12}$ $(\mathrm{F} / \mathrm{m})$, respectivamente.

\section{RESULTADOS Y DISCUSIÓN}

El modelo MEF desarrollado fue implementado en Matlab y para el cálculo del campo electromagnético alrededor de una línea eléctrica se presentan dos casos. El primero corresponde a una línea de transmisión trifásica de simple circuito, disposición horizontal, $500 \mathrm{kV}$, conductores ACSR $954 \mathrm{MCM}$, 
54/7, 995 A, en haz de cuatro conductores por fase, cable de guardia de acero galvanizado de $1 / 2$ ", Figura 2. El segundo caso corresponde a una línea de subtransmisión trifásica circuito simple, disposición vertical, $66 \mathrm{kV}$, conductores AA AWG 1, 177 A, Figura 3.

De la simulación de MEF llevada a cabo, se presenta la dispersión del campo eléctrico, primer caso, para

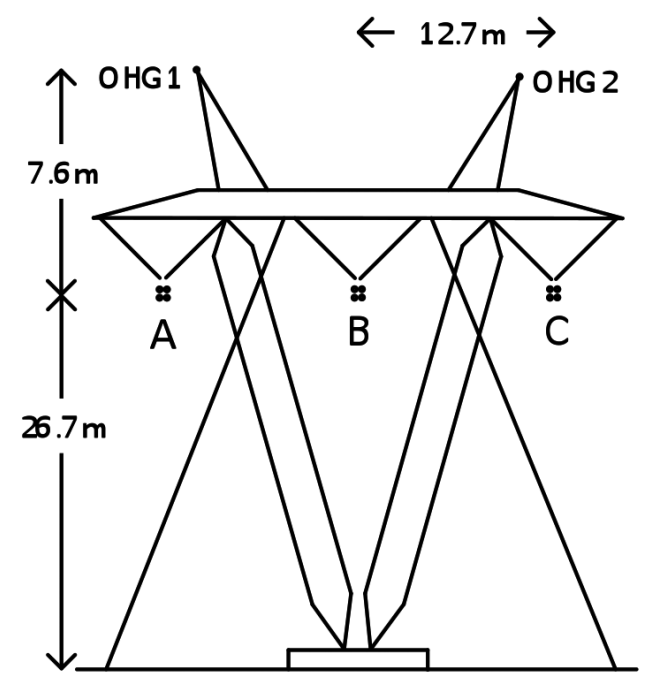

Figura 2. Línea de transmisión, $500 \mathrm{KV}$.

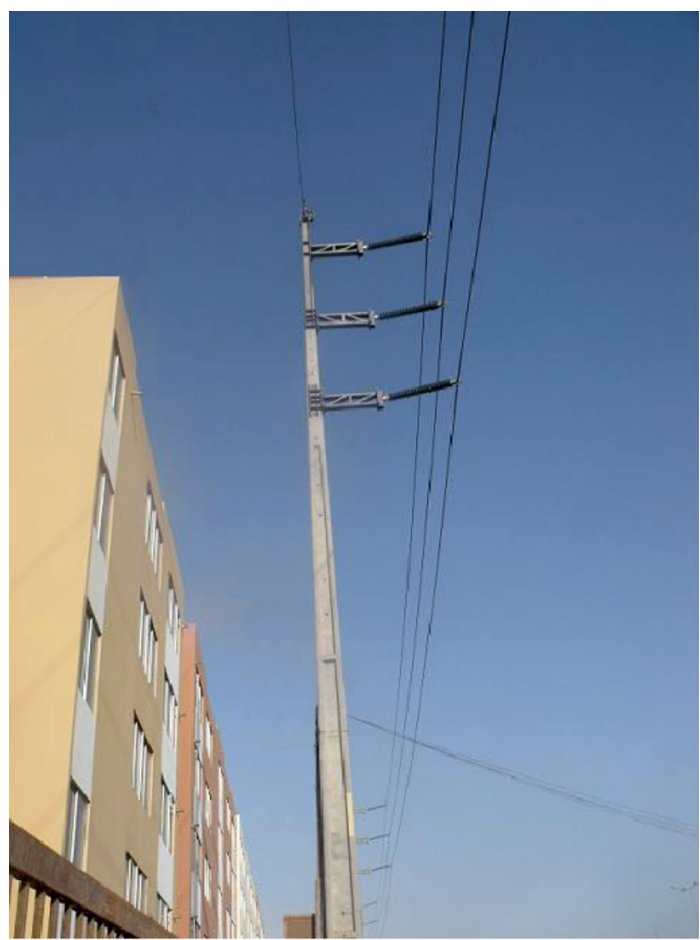

Figura 3. Línea de subtransmisión, $66 \mathrm{KV}$.

ello se emplea una característica gráfica de MATLAB que permite representar en áreas transversales las distintas magnitudes del campo magnético, Figura 4.

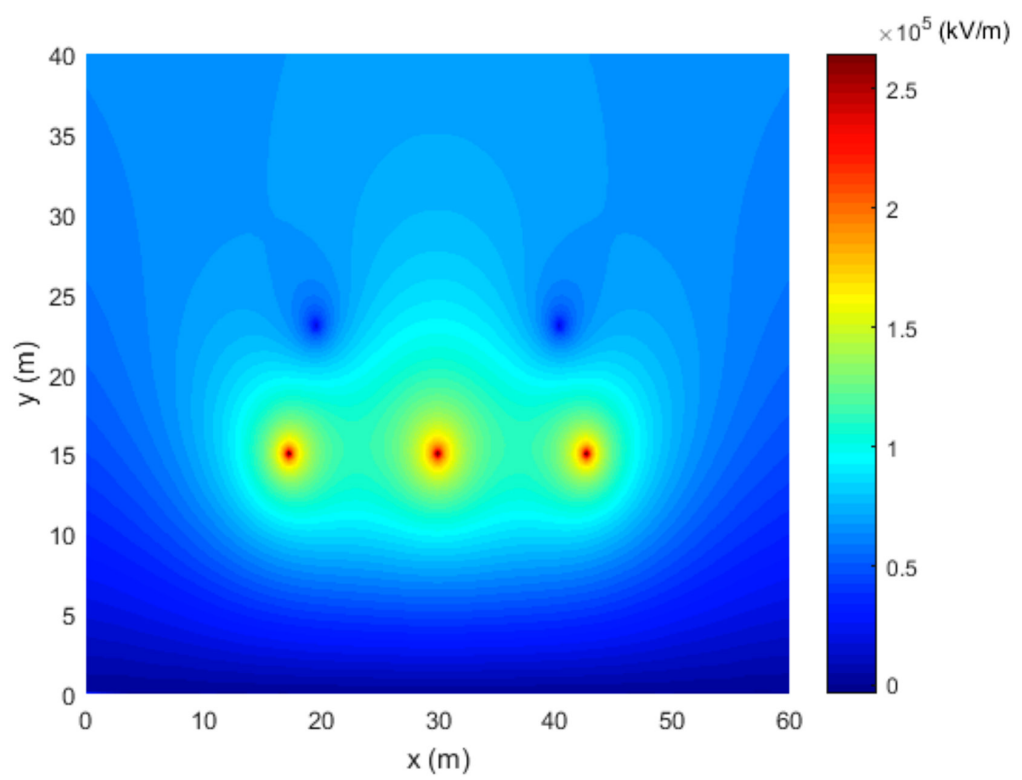

Figura 4. Distribución de la magnitud del campo eléctrico $(\mathrm{KV} / \mathrm{m})$, línea de $500 \mathrm{kV}$. 
Las distribuciones del campo eléctrico fueron simuladas bajo condiciones normales de carga, es decir, corrientes balanceadas en los conductores de fase. Para analizar los posibles efectos de la magnitud del campo eléctrico en humanos por debajo de las líneas de transmisión, se definen líneas de cálculo a 1, 2, 4, 6, 8 y 10 metros sobre el nivel del suelo. Los resultados comparativos del campo eléctrico en la línea de transmisión, se muestra en la Figura 5.

El segundo caso corresponde al de la línea de $66 \mathrm{kV}$. La particularidad de este estudio está en que el trazado de la línea se encuentra en una zona residencial, Figura 3. El estudio cobra relevancia por la extrema cercanía a un grupo de viviendas sociales. La distancia, entre los conductores de fase de la línea de transmisión y las viviendas sociales, se encuentra a menos de $4 \mathrm{~m}$.

La Figura 6, presenta en áreas transversales las distintas magnitudes del campo magnético.

Como una manera de cuantificar el campo magnético en zonas críticas, la Figura 7, presenta la magnitud del campo magnético en la vertical del edificio, a 4 y 6 metros de los conductores de fase.

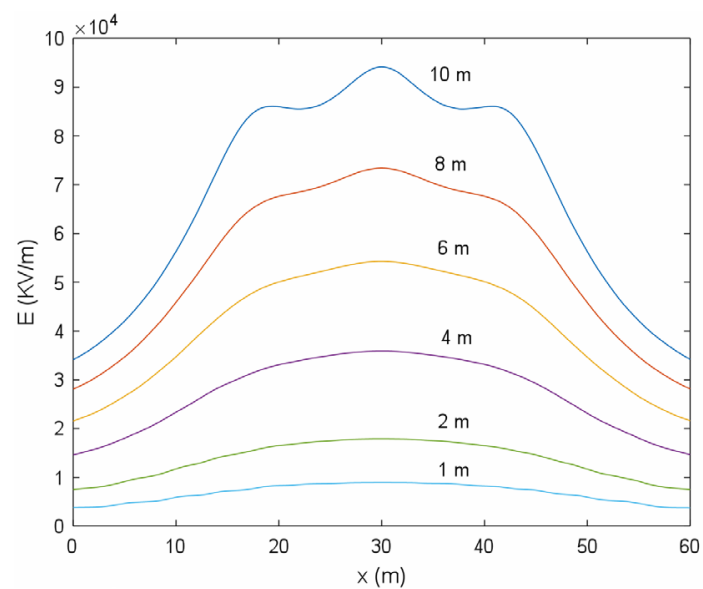

Figura 5. Magnitud del campo eléctrico (KV/m), a distintas alturas debajo de la línea de $500 \mathrm{kV}$.

Se observa que los niveles de magnitud máxima del campo magnético se encuentran entre los 8 a $12 \mathrm{~m}$ de altura, frente a los últimos pisos del edificio, a la altura de la posición de los conductores de fase. El método de análisis empleado arroja resultados que, si bien son altos, no sobrepasan los valores recomendados por las agencias regulatorias internacionales $[1,2$,

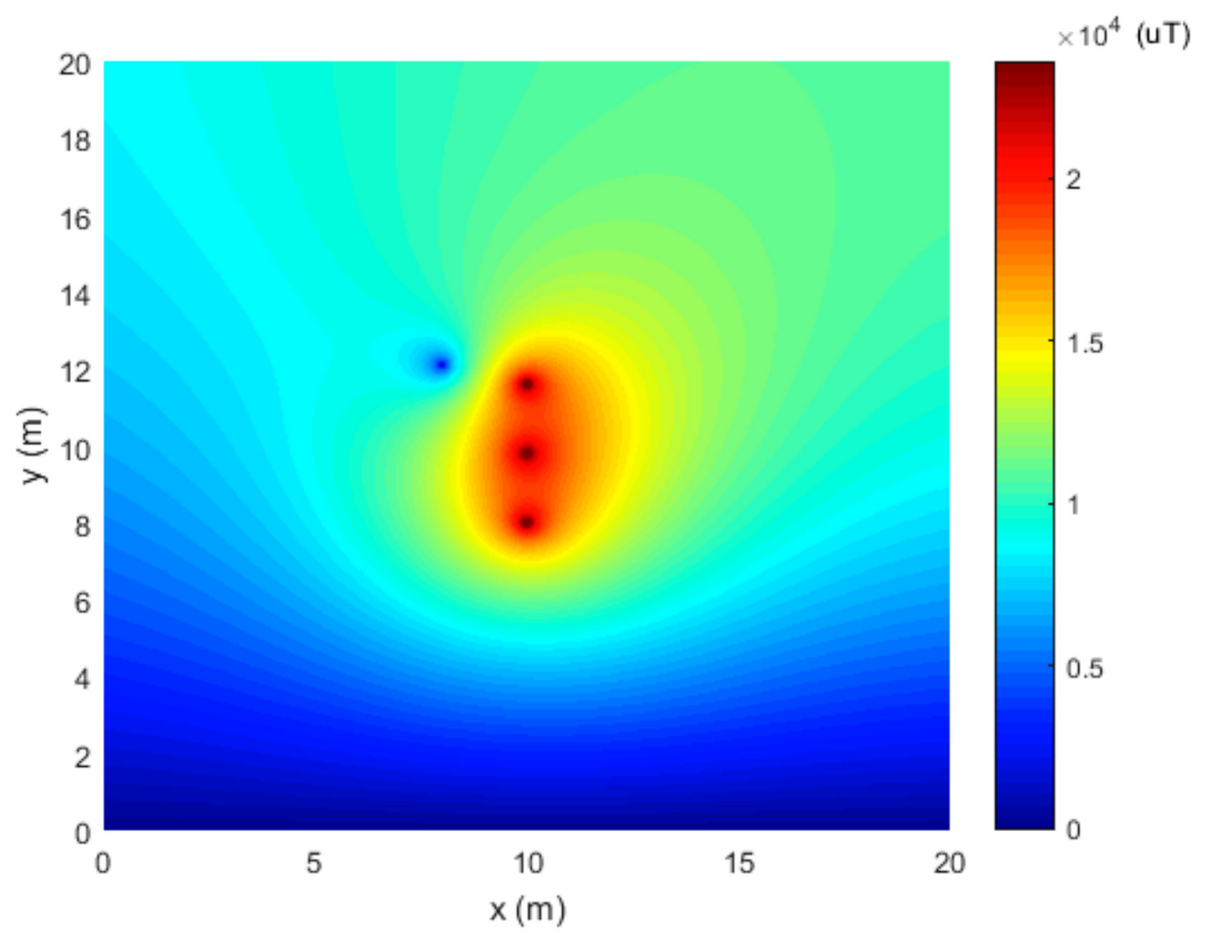

Figura 6. Distribución de la magnitud del campo magnético en ( $\mu \mathrm{T})$, línea de 66(KV). 


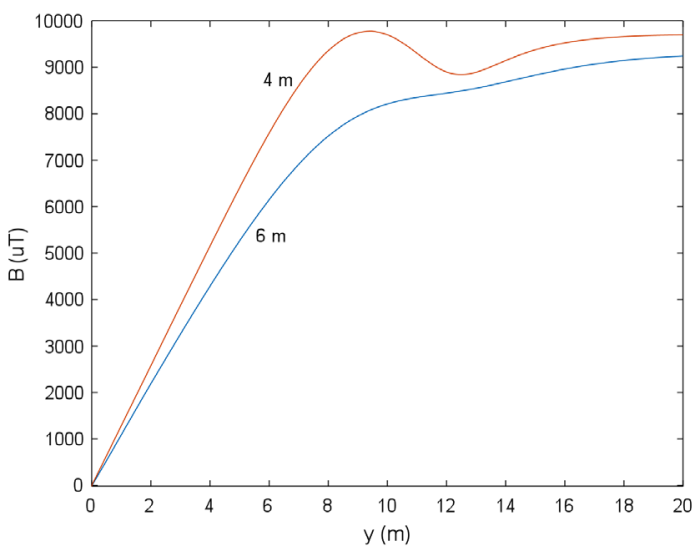

Figura 7. Magnitud del campo magnético en la vertical del edificio, a 4 y 6 metros de los conductores de fase.

3], dado su carácter de exposición permanente, son de cuidado para un recinto habitable.

\section{CONCLUSIONES}

En este trabajo se ha aplicado el método de elementos finitos para cuantificar el campo electromagnético que rodea a una línea de transmisión eléctrica, en condiciones normales de carga. Se investigaron dos casos; el primero corresponde a una línea de transmisión de 500 kV, en configuración horizontal, en circuito simple, correspondiente al nivel de voltaje más alto en Chile. El resultado en condición de carga normal, revela que el campo magnético a un nivel de $1 \mathrm{~m}$ sobre el suelo, que se supone que es el nivel de trabajo humano, no excede el máximo permitido cuando se contrasta con la norma internacional ICNIRP, [1]. El segundo caso, corresponde a una línea de $66 \mathrm{kV}$, en disposición vertical en simple circuito, cuyo trazado se encuentra en una zona residencial. En este caso, se investigó la magnitud del campo magnético sobre el edificio, frente a los conductores, que para la condición de carga normal, también está bajo el máximo permitido por norma. Por otra parte, es importante hacer notar, que la estructura metálica interna del edificio, actúa como un apantallamiento para el campo electromagnético, reduciendo su efecto al interior de éste. Este fenómeno, no está considerado en este estudio.

Las enfermedades que se pueden ocasionar con una exposición prolongada a campos electromagnéticos en baja frecuencia aún no han sido comprobadas científicamente; existen diversos estudios, donde se dan argumentos en contra y a favor sobre este tema $[2,3]$. Sin embargo, como principio de prevención, en la mayoría de los países se han adoptado normas que fijan niveles máximos de exposición para espacios públicos, mientras no se demuestre que los campos electromagnéticos no son dañinos para la salud.

\section{AGRADECIMIENTOS}

Los autores agradecen Dr. Kristopher Chandia por el aporte realizado en el trabajo, así como también, el apoyo de la Universidad de Tarapacá, proyecto 8723-06.

\section{REFERENCIAS}

[1] Salud Geoambiental. "Mapa de niveles de radiación electromagnética permitidos por la legislación de distintos países". Fecha de consulta: 25 de noviembre de 2020 . URL: https://www.saludgeoambiental.org/ legislacion-0

[2] J.P. Reilly. "Human exposure standards in the frequency range $1 \mathrm{~Hz}$ to $100 \mathrm{kHz}$ ". Health Physics. Vol. 107 N $^{\circ}$ 4, pp. 343-350. 2014.

[3] A. Hamza, S. Mohmoud and S. Ghania. "Environmental pollution by magnetic field associated with power transmission lines". Energy Conversion and Management. Vol. 43, pp. 2443-2452. 2002.

[4] K. Hameyer, R. Mertens and R. Belmans. "Numerical methods to evaluate the electromagnetic fields below overhead transmission lines and their measurement". Proceedings of First International Caracas Conference on Devices, Circuits and Systems, pp. 32-36. 1995.

[5] Y.W. Kwon and H. Bang. "The finite element method using matlab". CRC Press. 2000.

[6] J.M. Jin. "The finite element method in electromagnetics". Third Edition. Wiley. 2014. Hoboken, Estados Unidos.

[7] P. Pao-La-Or, A. Isaramongkolark and T. Kulworawanichpang. "Electro-magnetic field distribution of phase-sequence orientation of a double-circuit power transmission line based on finite element method". WSEAS Transactions on power systems. Vol. $3 \mathrm{~N}^{\mathrm{o}} 10$, pp. 653-663. 2008.

[8] P. DhanaLakshmi, L. Kalaivani and P. Subburaj. "Analysis of magnetic field 
distribution in power system using finite element method". International Conference on Computer, Communication and Electrical Technology - ICCCET2011. 18-19 Marzo 2011.

[9] S. Rojo. "Análisis de la distribución del campo eléctrico y magnético en líneas de transmisión eléctrica a través de la técnica de elementos finitos". Universidad de Tarapacá. 2011. Arica, Chile.
[10] M. Melo, L. Fonseca, E. Fontana and S. Naidu. "Electric and magnetic fields of compact transmission lines". IEEE Transactions on Power Delivery. Vol. 14 No 1, pp. 200-204. 1999.

[11] P-O. Person and G. Strang. "A simple mesh generator in MATLAB". SIAM Review. Vol. $46 \mathrm{~N}^{\circ}$ 2. Fecha de consulta: 25/11/2020. URL: https://github.com/ionhandshaker/ distmesh. 